\title{
WestVirginiaUniversity
}

THE RESEARCH REPOSITORY @ WVU

Graduate Theses, Dissertations, and Problem Reports

2006

\section{Propagation of Alfven waves in the WVU HELIX device}

Christopher S. Compton

West Virginia University

Follow this and additional works at: https://researchrepository.wvu.edu/etd

\section{Recommended Citation}

Compton, Christopher S., "Propagation of Alfven waves in the WVU HELIX device" (2006). Graduate Theses, Dissertations, and Problem Reports. 2450.

https://researchrepository.wvu.edu/etd/2450

This Thesis is protected by copyright and/or related rights. It has been brought to you by the The Research Repository @ WVU with permission from the rights-holder(s). You are free to use this Thesis in any way that is permitted by the copyright and related rights legislation that applies to your use. For other uses you must obtain permission from the rights-holder(s) directly, unless additional rights are indicated by a Creative Commons license in the record and/ or on the work itself. This Thesis has been accepted for inclusion in WVU Graduate Theses, Dissertations, and Problem Reports collection by an authorized administrator of The Research Repository @ WVU. For more information, please contact researchrepository@mail.wvu.edu. 
Propagation of Alfvén Waves in the WVU HELIX Device

\author{
Christopher S. Compton
}

\author{
Thesis submitted to the \\ Eberly College of Arts and Sciences \\ at West Virginia University \\ in partial fulfillment of the requirements \\ for the degree of
}

\author{
Master of Science \\ in \\ Physics
}

\author{
E.E. Scime, Ph.D., Chair \\ H. A. Weldon, Ph.D. \\ J. E. Littleton, Ph.D. \\ Department of Physics \\ Morgantown, West Virginia
}

Keywords: Alfvén Waves, Plasma Physics, Helicon Source 


\title{
ABSTRACT \\ Propagation of Alfvén Waves in the WVU HELIX Device
}

\section{Christopher S. Compton}

\begin{abstract}
Alfvén waves are low frequency, long wavelength electromagnetic waves commonly found in space plasmas. As these waves typically have wavelengths on the order of kilometers, generating Alfvén waves in the lab is quite difficult. At the appropriate plasma parameters, (e.g., high density and weak applied magnetic field strength), the wavelength of the Alfvén wave will be small enough to fit into a reasonably sized experimental device. Here it will be shown that Alfvén waves have been successfully propagated in the HELIX device in argon and helium plasmas.
\end{abstract}




\section{Acknowledgements}

I am deeply indebted to my advisor Dr. Earl Scime. I want to thank him for his patience and guidance along the way. Thanks also to Dr. Costel Biloiu for all of his advice in the lab. I was able to learn a great deal from him. Thanks to my fellow students: Amy, Xuan, Rob, and Ryan for all of your helpful discussions, suggestions and assistance. Thanks to Carl, Doug and Tom for their advice in the design of and construction of the probes and other experimental devices. Siobhan and Sherry were extremely helpful in helping me take care of all of the administrative requirements. I thank Amy for always being around to help me through frustrations and giving me a life outside the lab. I thank Rebecca and Phil for keeping me up to date with the music scene in Morgantown. Thanks to Sean and Maple for giving me a love and sense of belonging to West Virginia and WVU. I owe a great deal of thanks to my parents for their support through these many, many years. My Grandfather was and forever will be an inspiration to me. I can only hope to live to be the person he saw in me.

In loving memory, Peggy Hall (1934-2000)

H.E. Hall (1935-2004) 


\section{TABLE OF CONTENTS}

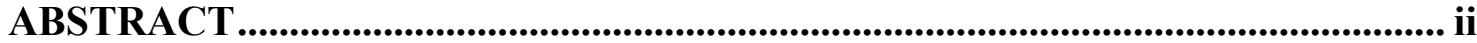

ACKNOWLEDGEMENTS ......................................................................................... ii

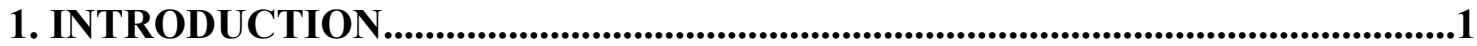

2. ALFVÉN WAVE DISPERSION RELATION .......................................................4

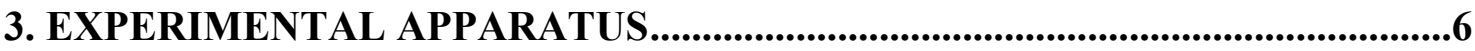

4. ALFVÉN WAVE MEASUREMENTS ...............................................................11

4.1 PRELIMINARY MEASUREMENTS ...................................................11

4.2 ARGON: FIXED FIELD, CHANGING DRIVE FREQUENCY .................13

4.3 HELIUM: CHANGING FIELD, FIXED DRIVE FREQUENCY ...............14

4.4 ARGON: FIXED FIELD, CHANGING DRIVE FREQUENCY .................18

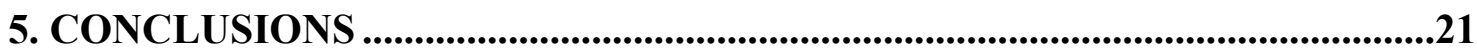

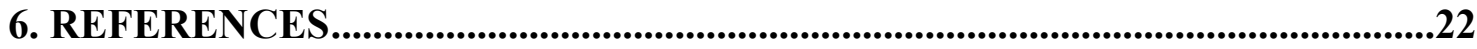




\section{INTRODUCTION}

Alfvén waves were first predicted by Hannes Alfvén in 1942 [Alfvén, 1942]. The classic Alfvén wave is a cold plasma wave with frequency much less than the cyclotron frequency, and follows the dispersion relation given by,

$$
\left(\omega / k_{\|}\right)^{2}=v_{A}^{2}\left(1-\omega^{2} / \omega_{c i}^{2}+k_{\perp}^{2} \rho_{s}^{2}\right),
$$

where $k$ is the parallel or perpendicular wave number relative to the magnetic field, $v_{\mathrm{A}}=\frac{B}{\sqrt{\mu_{0} n m_{i}}}$ is the Alfvén speed, $\omega_{c i}$ is the ion cyclotron frequency, and $\rho_{s}=\frac{\sqrt{T_{e} / m_{i}}}{\omega_{c i}}$ is the ion sound gyroradius. Since Alfvén waves only exist below the ion cyclotron frequency, they generally have a long wavelength. Thus, Alfvén waves propagate more easily in unbounded space plasmas than in spatially limited laboratory plasmas. The first laboratory experiment to demonstrate Alfvén wave propagation was performed in mercury by Lundquist [Lundquist 1949]. Three years later, in 1952, the first laboratory evidence of Alfvén waves in an ionized gas was reported by Bostick and Levine [Bostick and Levine, 1952]. Their experiments were performed in a toroidal tube filled with helium gas at a pressure of 20 mTorr and a plasma density of about $5 \times 10^{14} \mathrm{~cm}^{-3}$. The Alfvén waves were detected at about 10 kilohertz using a Langmuir probe.

Since then, Alfvén waves have been observed in association with a variety of natural and manmade plasma phenomena. Alfvén waves are routinely observed in planetary magnetospheres and coronal holes [Marsch, 1991; Goldstein et al., 1995; Bloomberg and Cummock, 2004] and as a result of reconnection events [Chaston et al., 2005]. For example, in the Earth's magnetopause, drift kinetic and kinetic Alfvén waves were recently observed to propagate away from a reconnection X-point by the Cluster 
spacecraft [Chaston et al., 2005]. In one rather unusual circumstance, Alfvén waves were produced following the detonation of an atomic bomb over the south pacific in 1962. Electric currents induced in a power grid in Hawaii by the electric fields of the Alfvén wave caused severe damage [Colgate, 1965; Gekelman, 2003]. In the laboratory, a laser produced plasma has been shown to produce Alfvén waves due to the rapid expansion of the plasma during the laser pulse [Gekelman, 2003]. In more conventional laboratory experiments, Alfvén waves are produced using coil antennae [Hanna and Watts, 2001], wire grids [Gekelman, 1994], or even the cathode-ground plane cavity of a high power pulsed plasma device [Maggs and Morales, 2003].

It has been theorized that ion heating by Alfvén waves may be sufficient to explain the large ion temperature gradient seen in the solar corona [Mattheus, 1999]. The ion heating is thought to be driven by the interaction between upwardly propagating Alfvén waves and Alfvén waves reflecting off the gradient in the Alfvén speed resulting from the density and magnetic field gradients in the expanding solar corona. The upward propagating wave and the reflected waves interact nonlinearly and drive MHD turbulence that then heats the ions. While there are difficulties in testing such an all-encompassing theory, laboratory experiments can be developed to try to investigate specific aspects of this hypothesis. The HELIX/LEIA device has similar parameters to that of the solar corona. The magnetic field geometry, plasma beta, and Alfvén speed gradient in HELIX/LEIA are all comparable to coronal values. To drive nonlinear interactions between the Alfvén waves, theory suggests that it is necessary to produce a perturbed magnetic field that is $5 \%$ of the background field, equivalent to a perturbation of about 50 Gauss for typical HELIX parameters. The critical first step in testing any theory of ion 
heating by Alfvén waves is to be able to reliably launch Alfvén waves in the appropriate experimental conditions. Currently groups at WVU and UCLA are pursuing this area of research.

Research on Alfvén wave propagation in helicon plasmas has only recently been published [Hanna and Watts, 1999; Grulke et al., 2003]. The helicon source offers an attractive solution for laboratory studies of the Alfvén waves owing to the ease of high density plasma production in these devices. The Hanna and Watts experiments at Auburn University were able to show that Alfvén waves could propagate in a helicon plasma [Hanna and Watts, 1999]. In their experiment, an Alfvén speed of $3.4 \times 10^{5} \mathrm{~m} / \mathrm{s}$ and wavelength of just a few meters was measured. Similar experiments carried out on VINETA at the Max Plank Institute found parallel wavelengths of 2-10 meters with an Alfvén speed of $4.5 \times 10^{5} \mathrm{~m} / \mathrm{s}$ [Grulke et al. 2003].

To confirm that Alfvén waves are being launched, one must examine the properties of the wave and confirm that they match theoretical predictions. In this thesis, the dispersion relation is derived and compared to the simple relation given in Eq. (1); the experimental apparatus constructed to launch Alfvén waves is described; and preliminary measurements of waves excited in helicon plasmas are compared to the predictions for Alfvén wave propagation. 


\section{ALFVÉN WAVE DISPERSION RELATION}

The HELIX device is capable of operating in both the inertial $\left(v_{e} / v_{A}\right)^{2}<1$, and the kinetic $\left(v_{e} / v_{A}\right)^{2}>1$ regimes for Alfvén waves (where $v_{e}=\sqrt{\frac{s_{e}}{m_{e}}}$ is the electron thermal speed), so it is necessary to derive the dispersion relation for both cases. From the plasma dielectric tensor and Maxwell's equations Boyd and Sanders [Boyd and Sanders, 2003] derive the general Alfvén dispersion relation

$$
\left[\frac{\omega^{2}}{k^{2}}-v_{A}^{2} \cos ^{2} \theta\right]\left[\frac{\omega^{4}}{k^{4}}-\frac{\omega^{2}}{k^{2}}\left(a^{2}+v_{A}^{2}\right)+a^{2} v_{A}^{2} \cos ^{2} \theta\right]=\Omega_{i}^{2} v_{A}^{4}\left(\frac{\omega^{2}}{k^{2}}-a^{2}\right) \cos ^{2} \theta
$$

where $a=\sqrt{T_{e} / m_{i}}$ is the sound speed, and $\Omega_{i}=\omega / \omega_{c i}$ and $\theta$ is the angle of the wave vector relative to the background magnetic field. Shear, transverse, Alfvén waves will be launched in the experiments described in this work, so the equations must be reduced to the shear case. Using an approach outlined by Cross [1988], we first rearrange the equation in powers of $k$ and using $k_{\|}=k \cos \theta$ we obtain

$$
k^{4}\left[\frac{\Omega_{i}^{2}\left(\frac{\omega^{2}}{k^{2}}-a^{2}\right)}{1-\frac{\omega^{2}}{v_{A}^{2} k_{\|}^{2}}}\right]+k^{2}\left[a^{2} k_{\|}^{2}-\omega^{2}\left(1+\frac{a^{2}}{v_{A}^{2}}\right)\right]+\frac{\omega^{4}}{v_{A}^{4}}=0
$$

Taking the last term to be small (since $v_{A}$ is assumed to be large) results in 


$$
k^{2}=-\frac{a^{2} k_{\|}^{2}-\omega^{2}\left(1+\frac{a^{2}}{v_{A}^{2}}\right)}{\Omega_{i}^{2}\left(\frac{\omega^{2}}{k^{2}}-a^{2}\right)}\left(1-\frac{\omega^{2}}{v_{A}^{2} k_{\|}^{2}}\right) .
$$

Rewriting in terms of $\omega$

$$
\omega^{2}\left[-\Omega_{i}^{2}\left(1-\frac{a^{2} k^{2}}{\omega^{2}}\right)+1+2 \frac{a^{2}}{v_{A}^{2}}-\frac{\omega^{2}}{v_{A}^{2} k_{\|}^{2}}\left(1+\frac{a^{2}}{v_{A}^{2}}\right)\right]=a^{2} k_{\|}^{2}
$$

Since we are looking at shear Alfvén waves, the perturbation will be perpendicular to the magnetic field and the wave will travel along the axis of the experiment. The maximum wavelength of the Alfvén wave is limited by the size of the experiment. Since the chamber is much larger in length than it is in radius, we may further approximate this expression by taking $k_{\|} \ll k_{\perp}$ and using $k^{2}=k_{\perp}^{2}+k_{\|}^{2}$. Since $a \ll v_{A}$ we can ignore the $a^{2} / v_{A}^{2}$ terms as well as the right hand side of Eq. (5). Thus,

$$
\frac{\omega^{2}}{v_{A}^{2} k_{\|}^{2}}=1-\Omega_{i}^{2}\left(1-\frac{a^{2} k_{\perp}^{2}}{\omega^{2}}\right)
$$

or, in a form that more clearly shows the ion cyclotron resonance,

$$
\frac{\omega^{2}}{v_{A}^{2} k_{\|}^{2}}=1-\frac{\omega^{2}}{\omega_{c i}^{2}}+\rho_{s}^{2} k_{\perp}^{2}
$$

where, $\rho_{s}=a / \omega_{c i}$. For sufficiently low temperature plasma, the sound term can be ignored leaving

$$
\frac{\omega^{2}}{v_{A}^{2} k_{\|}^{2}}=1-\frac{\omega}{\omega_{c i}}
$$

which is the form obtained for the classic inertial Alfvén wave. 
Alfvén waves in the inertial regime, where $k_{\perp} \rho_{s} \ll 1$, propagate in a cone relative to the magnetic field as described by Gekelman et al. [1994]. The angle at which the propagation occurs is given by $\phi=\tan ^{-1} \mathrm{k}_{\mathrm{A}} \delta$, where $k_{\mathrm{A}}$ is the Alfvén wave number and $\delta$ is the electron plasma skin depth, $\delta=c / \omega_{p e}$. At some critical axial distance, $z=\frac{r}{\tan \theta}$, the cone will become hollow and the wave power will be seen to propagate along the outer edge of the cone. In this thesis, low power (small amplitude) Alfvén waves will be shown to be reliably launched and received in the WVU HELIX device. The waves propagate at the expected cone angle relative to the magnetic field and their dispersion relation is consistent with the theoretical predictions.

\section{EXPERIMENTAL APPARATUS}

These experiments were performed in the HELIX (Hot hELIcon eXperiment) device. The HELIX device, shown in Figure 1, consists of a $10 \mathrm{~cm}$ OD Pyrex ${ }^{\mathrm{TM}}$ tube connected to a $15.25 \mathrm{~cm}$ OD stainless steel tube. The two chambers have a total length of $1.57 \mathrm{~m}$. The plasma is generated by a $19 \mathrm{~cm}$ helical antenna that is mounted around the Pyrex ${ }^{\mathrm{TM}}$ section of the chamber. There are 10 electromagnets surrounding HELIX. These coils are each made of 46 copper windings and all connected in series to produce a magnetic field of up to 1300 Gauss. HELIX is connected to LEIA which is a $2 \mathrm{~m}$ OD, 4.5 m long aluminum chamber. LEIA has seven electromagnets made of aluminum tubing, each with 40 turns. In these experiments, the magnetic field in LEIA was not used and the plasma was allowed to freely expand into LEIA. In trying to find the best conditions for matching the plasma, it was found that having no field in LEIA produced the most stable 
helicon mode in helium. Base pressures in the HELIX-LEIA device reach $8 \times 10^{-8}$ Torr through the use of three turbomolecular drag pumps.

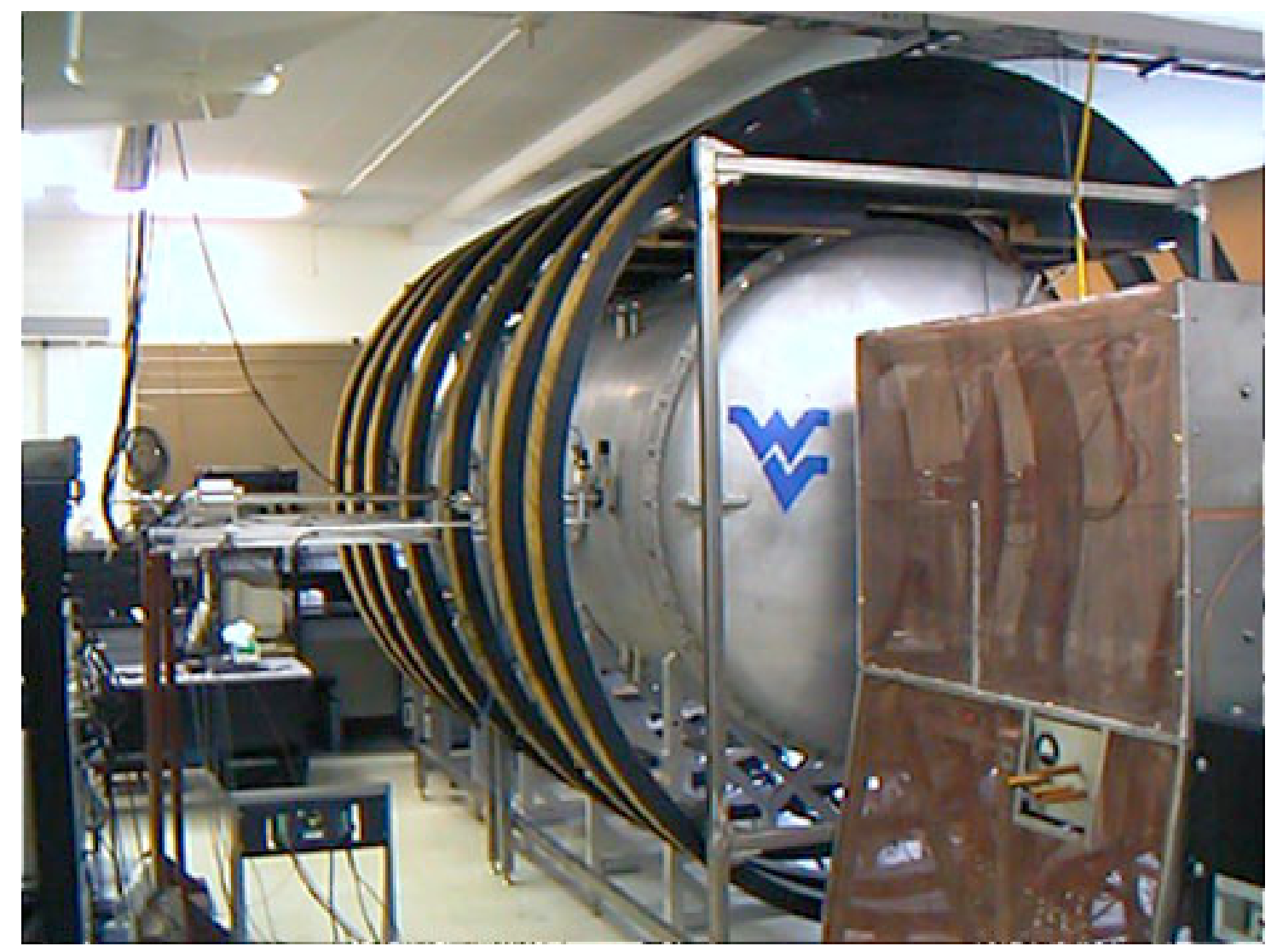

Figure 1. HELIX (foreground) and LEIA (background)

Alfvén waves were excited and detected with loop antenna probes. The transmitting coil is made from two $1.27 \mathrm{~cm}$ long ferrite rods glued end to end and wrapped with 60 turns of 32 gauge copper wire. The resistance of the transmitting coil was approximately $1-2$ Ohms. The receiving coil consists of a $1.27 \mathrm{~cm}$ ferrite core wrapped with 200 turns of 40 gauge copper wire. Receiving coils typically had a resistance of about $20 \mathrm{Ohms}$.

To make the coils capable of surviving for long periods of insertion in the plasma, the coils had to be cooled. Probe shafts were designed such that water could be 
introduced as a means of passively cooling the coils. This passive cooling scheme differs from the active system developed by Hanna and Watts [2001]. In their design, water was continuously cycled in and out of a glass shaft that held the coil.

The probe shafts used in the experiments reported here are made of stainless steel and are $40.5 \mathrm{~cm}$ long. The OD is $1.27 \mathrm{~cm}$ for the length of the shaft except the final $5 \mathrm{~cm}$ which has an $\mathrm{OD}$ of $0.89 \mathrm{~cm}$ as shown in Figure 2, where dimensions for the engineering schematic are presented in inches. The smaller OD at the end of the shaft is to help minimize the perturbation to the plasma due to the insertion of the conductor, and to reduce the attenuation of the signal. The ID is $0.635 \mathrm{~cm}$ for the entire length of the shaft. In future experiments, it is expected that the probe shafts will be coated with ceramic to further reduce perturbation to the plasma and the heat load on the coils.

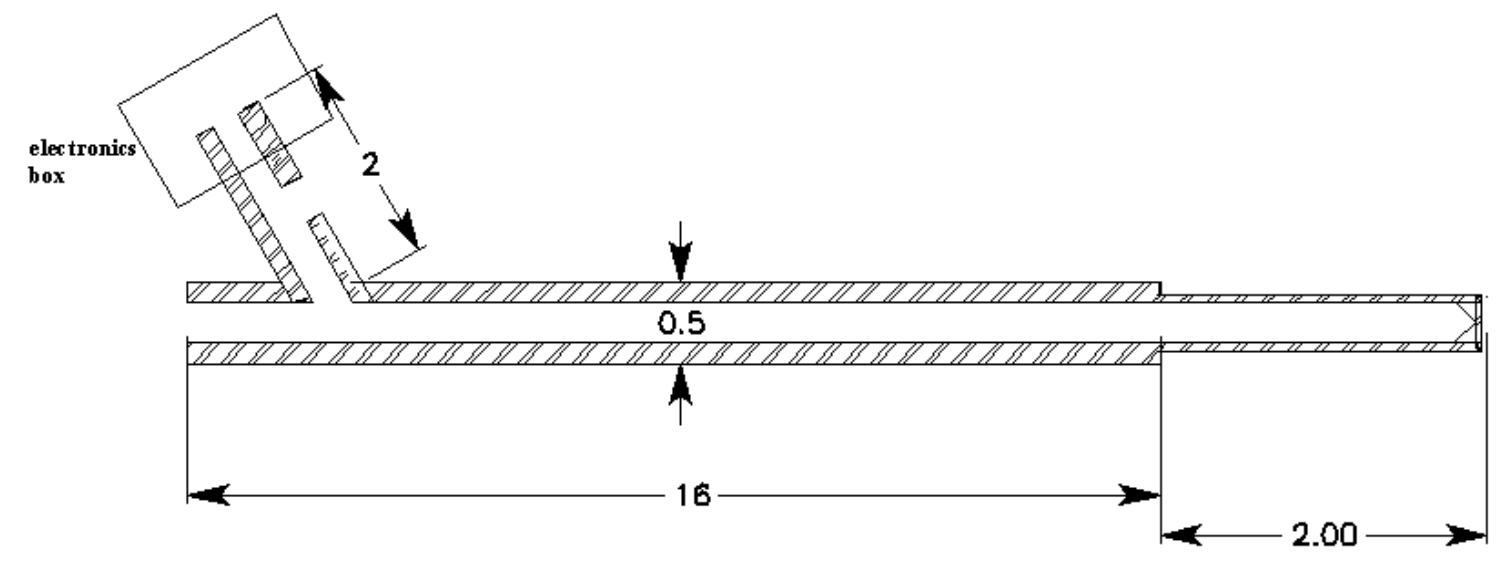

Figure 2. Engineering schematic of the probe shaft with dimensions in inches.

At the frequencies used to excite the Alfvén waves (19 kHz to $112 \mathrm{kHz}$ ), the skin depth $\Delta=\sqrt{2 \sigma / \mu_{0} \omega}$ (where $\sigma=$ conductivity) of stainless steel is $0.1-0.3 \mathrm{~cm}$. Some attenuation is expected since the thickness of the probe shaft at the coil location is 0.25 $\mathrm{cm}$. The coils are introduced into the probe shaft via a stainless steel strip that is bent at 
the end such that it creates a pocket for the coil to be cradled as it is inserted or removed. Passive water cooling was accomplished by welding a short section of tubing at a $60^{\circ}$ angle onto the main shaft. Water is added through a hole in the side of the short section of tubing and left open to air to allow heated water to evaporate. A plug is placed at the open end of the main shaft to allow the water to pool throughout the shaft. An aluminum box in which is mounted a differential BNC connector is attached to the short section of tubing and the antenna coil leads soldered to the BNC connector. The probes were inserted $30.5 \mathrm{~cm}$ apart in the stainless steel section of HELIX, as shown in Figure 4.

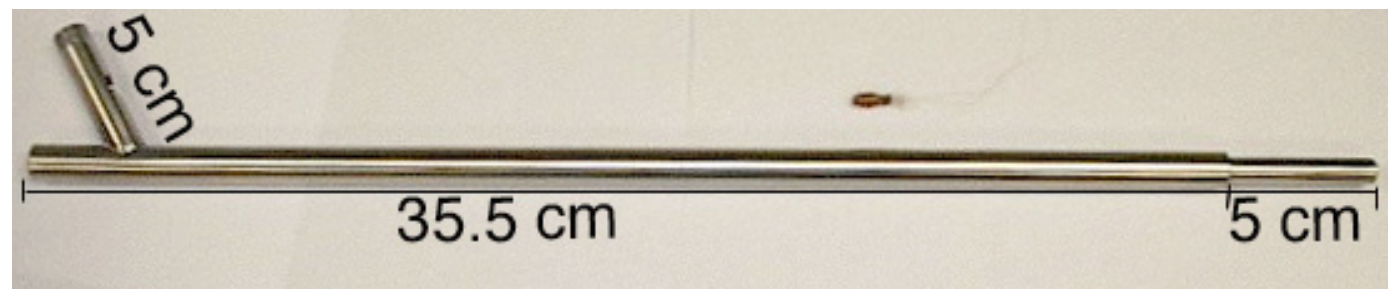

Figure 3. Probe shaft shown without electronics box, the port for introducing water on the left. A typical receiving coil is shown above the probe shaft.

The launching coil was connected via a RG58 coaxial cable to a Hewlett Packard 33120 function generator. The signal from the detector coil was too weak to be detected without amplification, so a variety of amplification methods were explored in order to improve the signal to noise of the received signal. In one set of experiments, a bandpass amplifier centered on $112 \mathrm{kHz}$ was employed. This amplifier had a gain of 50, but required the use of a single excitation frequency. In later experiments, a differential voltage amplifier with a wide frequency response $(1-40 \mathrm{kHz})$ and a selectable gain of 1/10 to 500 , designed and built by the University of Wisconsin-Madison plasma group, was used in series with the coil and oscilloscope. A RG-22 twin-axial cable was used 
from the detector coil to the differential amplifier. Because the Alfvén wave was localized radially to only a few $\mathrm{mm}$, initial detection of the propagating wave was accomplished by monitoring the amplifier signal on an oscilloscope while moving the detector probe radially. Once the signal was maximized, a lock-in amplifier was used to obtain phase measurements between the launching antenna and the detector.

Experiments were performed in both helium and in argon plasmas. This was done to accommodate other plasma diagnostics, such as laser induced fluorescence, that required a specific plasma species. Fill pressures in argon were 8-9 mTorr actual pressure. Helium fill pressures were 10-11 mTorr, actual pressure. For both plasma species, the magnetic field strength varied from 300-800 Gauss.

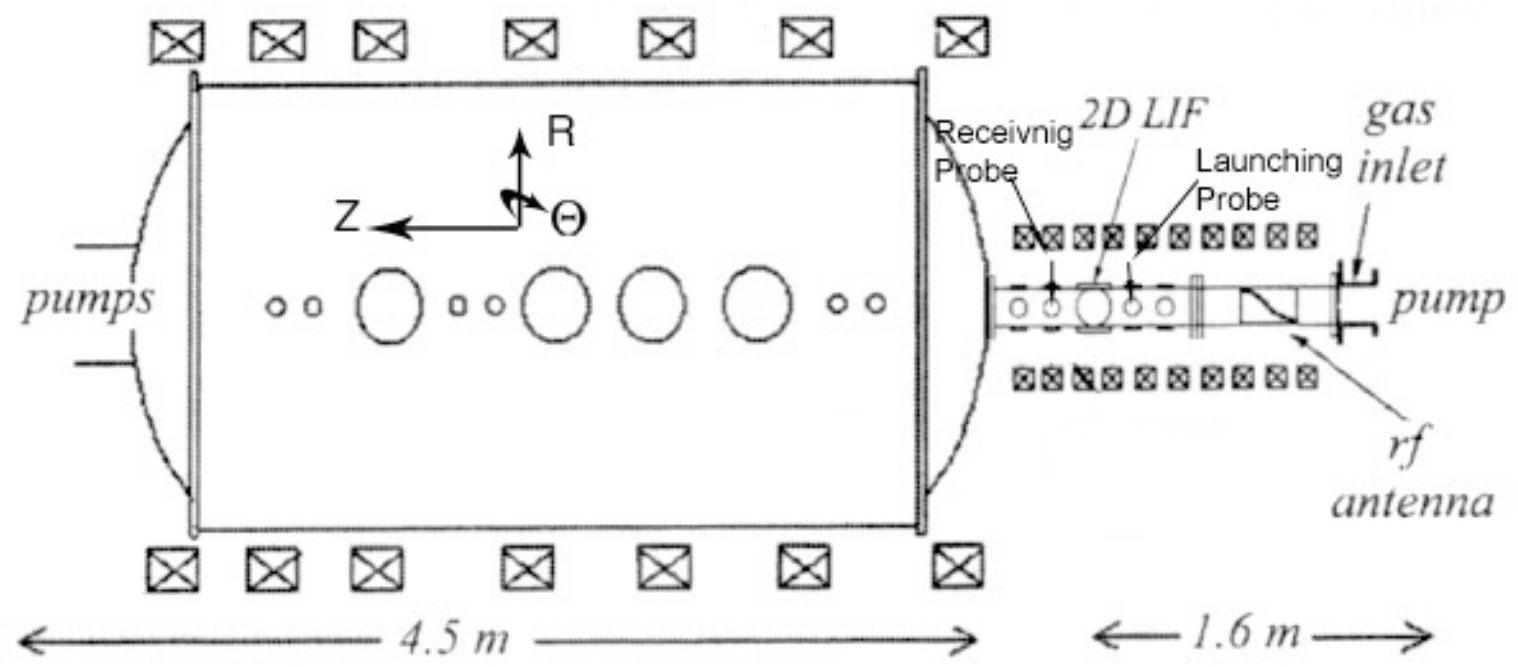

Figure 4. Experimental layout of the HELIX/LEIA device. The launching and receiving probes are shown in HELIX. 


\section{ALFVÉN WAVE MEASUREMENTS}

\subsection{PRELIMINARY MEASUREMENTS}

Preliminary proof-of-principal experiments were performed in argon plasmas at a RF power of 750 Watts, a background magnetic field strength of 770 Gauss, and a neutral gas pressure of 8.8 mTorr. Waves were launched in HELIX with a transmitting coil at 27 $\mathrm{kHz}$ and detected $1 \mathrm{~m}$ downstream in LEIA with a three-axis B-dot coil array (not the detection coil described previously) [Scime et al., 2002].

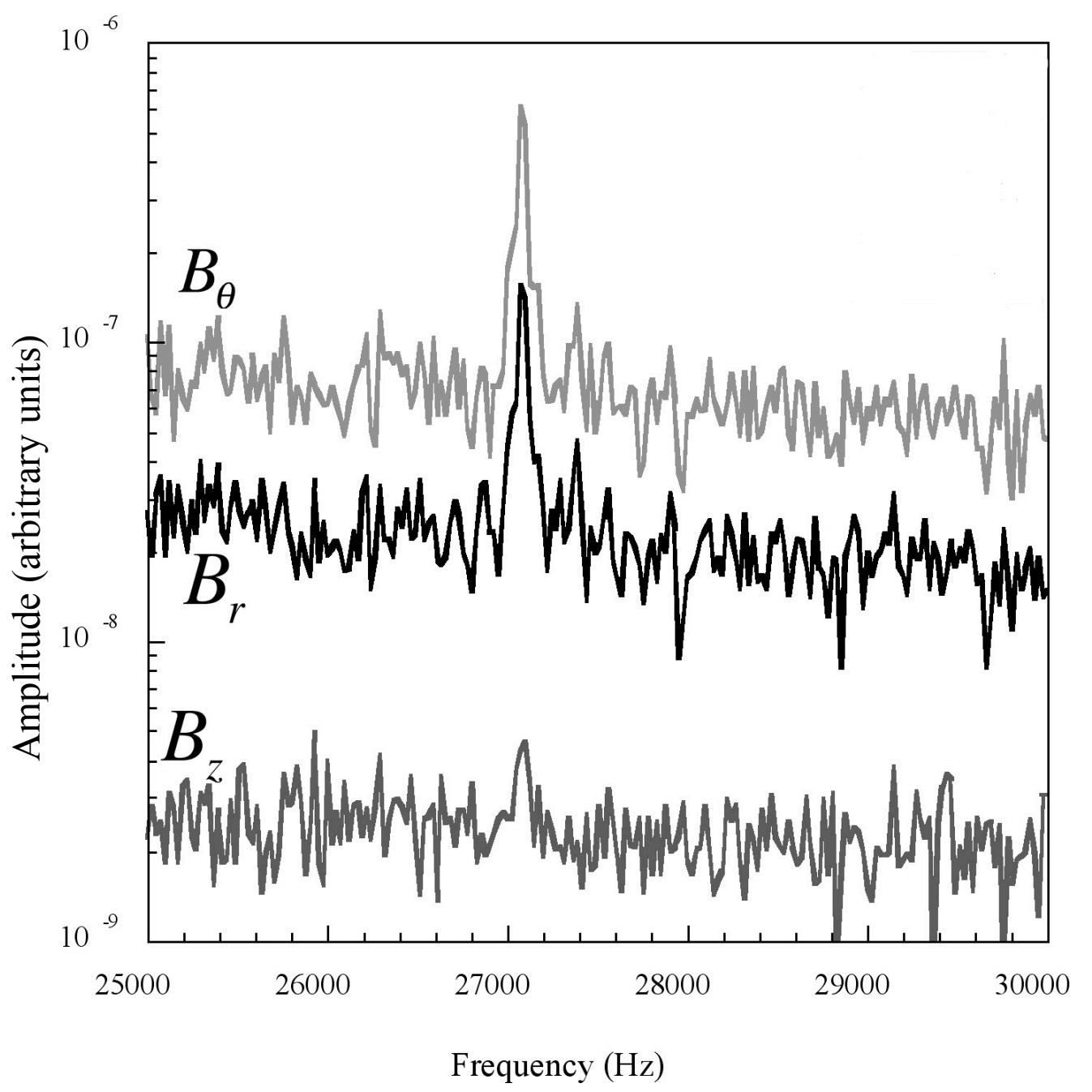

Figure 5. Three components of the magnetic field fluctuations measured in LEIA for wave excitation in HELIX. The perturbed magnetic field is primarily in the transverse direction. 
As shown in Fig. 5, well-defined, transverse waves were observed in LEIA at 27 $\mathrm{kHz}$. In HELIX, the wave frequency is subcyclotronic $\left(\omega / \Omega_{\mathrm{ci}}=0.92\right)$. Detection of the waves in LEIA was problematic. At launching frequencies other than the frequency that yielded the maximum power transfer to the transmitting coil, i.e, the frequency at which the antenna was impedance matched to the function generator, it was not possible to obtain reasonable signal-to-noise levels in LEIA. Another troublesome condition was that the power output of the function generator varied significantly with wave frequency due to better coupling with the antenna at higher frequencies. To confirm Alfvén wave excitation by measuring the wave dispersion relation as a function of wave frequency, measurements of the phase shift between launch and detection locations are required at multiple wave frequencies. Because signal detection in LEIA proved so problematic, the excitation and detection coil pair described in the previous section were used for the remainder of the measurements reported in this work. 


\subsection{ARGON: FIXED FIELD, CHANGING DRIVE FREQUENCY}

To improve the coupling of the signal generator to the transmitting coil, we experimented with the introduction of a Pi-matching network with a 1:1 center tapped transformer at the transmitting coil (shown in Figure 6). To raise the cyclotron frequency, and so that the driving frequency could be increased, the magnetic field was raised to 1069 Gauss and the source operated at a pressure of 14.7 mTorr of argon. The dispersion relation shown in Fig. 7 was obtained by placing the detection coil $30.5 \mathrm{~cm}$ downstream of the transmitting coil in HELIX and measuring the phase difference between the launched and received signals using a $200 \mathrm{kHz}$ digitizer and $\mathrm{PC}$ in lieu of the aforementioned amplifiers. Consistent with expectations for a shear Alfvén wave, as the ratio of launched frequency to the ion cyclotron frequency approached unity a sharp increase in the phase was observed. The corresponding parallel wavelength was approximately $6 \mathrm{~m}$, shorter than the $7 \mathrm{~m}$ long chamber. The plasma density calculated from the measured Alfvén speed of $2.4 \times 10^{5} \mathrm{~m} / \mathrm{s}$ agreed with previous plasma density measurements at a field strength of 1069 Gauss.

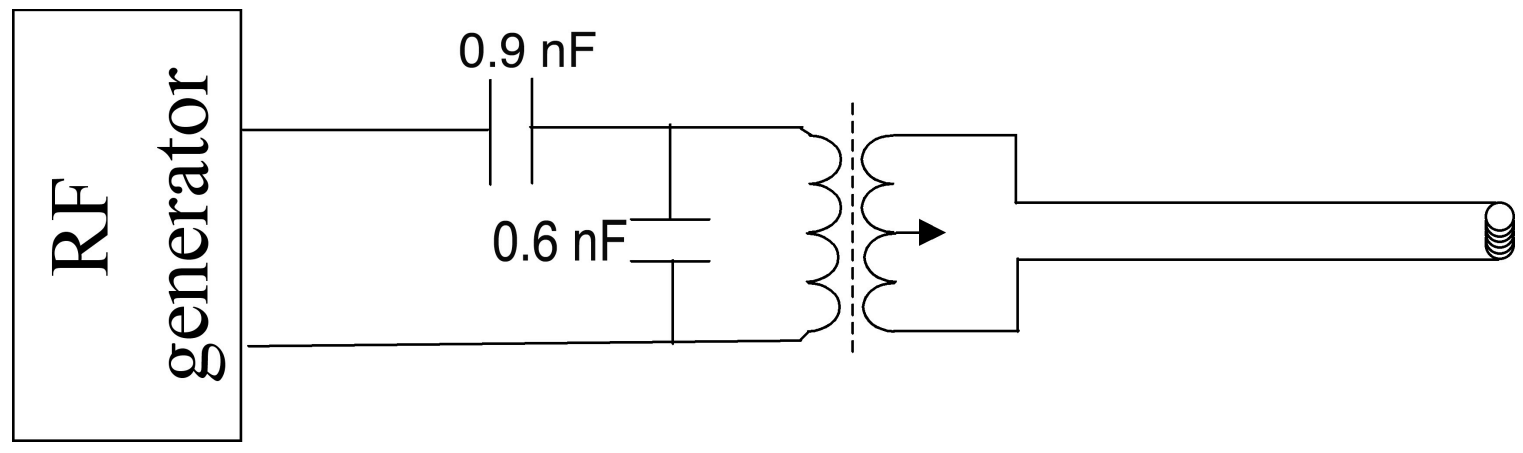

Figure 6. Schematic of transmitting coil and matching network. 


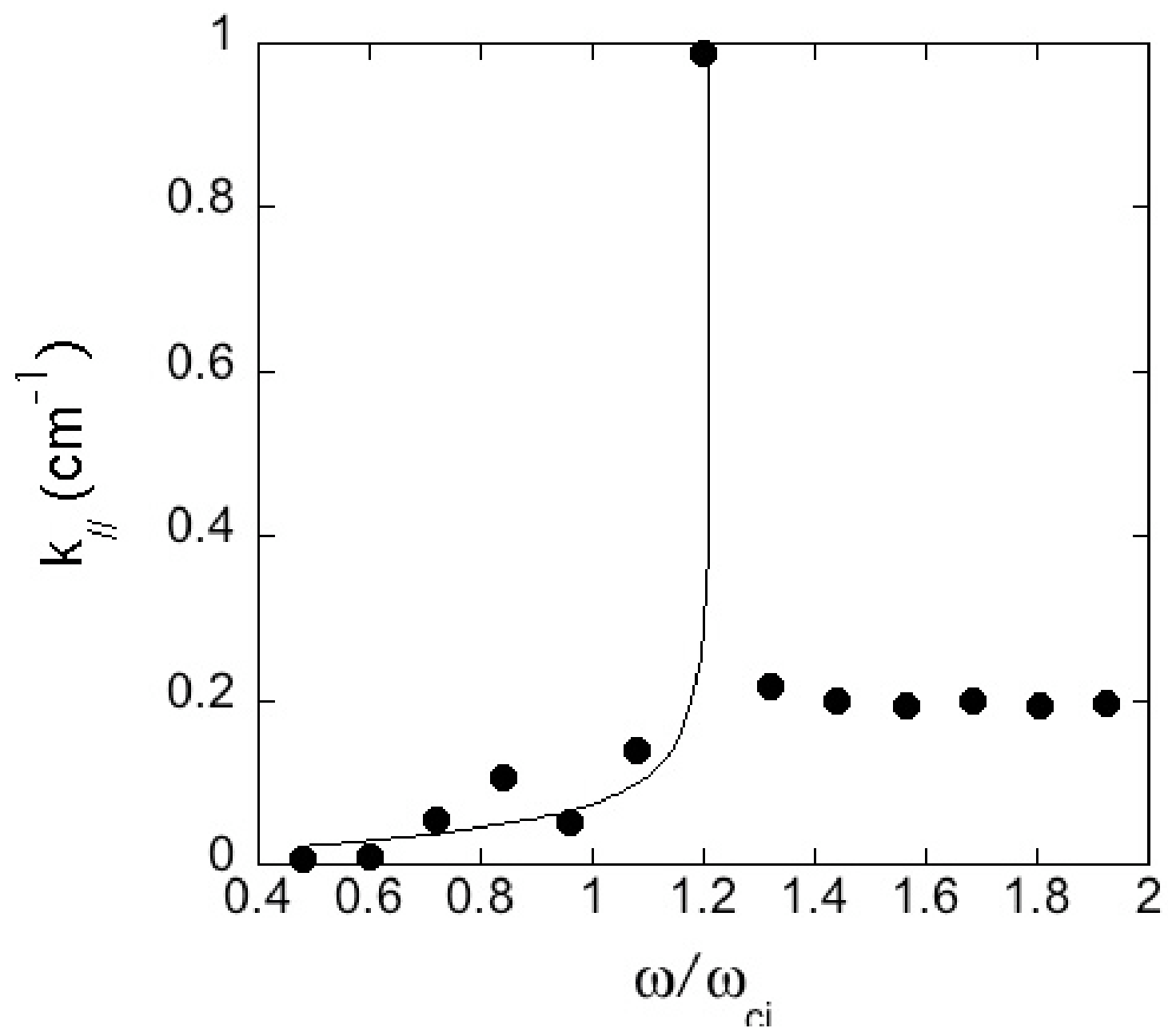

Figure 7. Parallel wave number versus normalized wave frequency $30.5 \mathrm{~cm}$ downstream in an argon HELIX plasma using a pi-matching network at the launching transmitter. Each phase (wave number) measurement was obtained at different excitation frequencies for a fixed value of the magnetic field strength.

\subsection{HELIUM: CHANGING FIELD, FIXED DRIVE FREQUENCY}

In a later experiment, to avoid the power variation in the transmitted signal due to the improved coupling to the antenna at higher frequencies, a fixed launching frequency was used while the magnetic field strength was varied. Here, as in the previous measurements, we measured the phase difference between the transmitting and receiving 
coils as a function of the ratio of the launching frequency of the wave to the ion cyclotron frequency. These experiments were performed in helium, allowing the use of a higher launching frequency since the ion cyclotron frequency of helium is higher than that of argon. Use of a higher excitation frequency is important because the function generator couples better to the antenna as the length of the launched wave is shortened. In these experiments, the signal from the receiver coil was passed through a $50 \mathrm{Ohm}$ resistor and a bandpass amplifier centered at $112 \mathrm{kHz}$ that had a gain of 50. Signal was first detected on the oscilloscope by triggering from a reference signal provided by the transmitter and averaging 30 measurements from the receiver to improve the signal to noise ratio.

The spatial distribution of the Alfvén wave, shown in Figure 8, was determined using the oscilloscope measurements and was found to have a full width at half maximum of $2.2 \mathrm{~cm}$, which agrees with the expected value obtained from theory derived for the Alfvén cone angle. The expected axial distance at which the Alfvén cone will become hollow is much longer than the probe separation; therefore the profile shown is expected to be centered about 0 . The radial profile shown in Fig. 8 was obtained while holding the plasma at a constant RF power of 450 Watts, gas pressure of 15.9 mTorr and background magnetic field of 600 Gauss. The measured Alfvén wave amplitude was 2 mGauss at the receiver location, $3 \times 10^{-4} \%$ of the background magnetic field. The magnetic field strength was determined from the voltage induced in the coil, the resistance of the coil and known equations for determining magnetic field strength. 


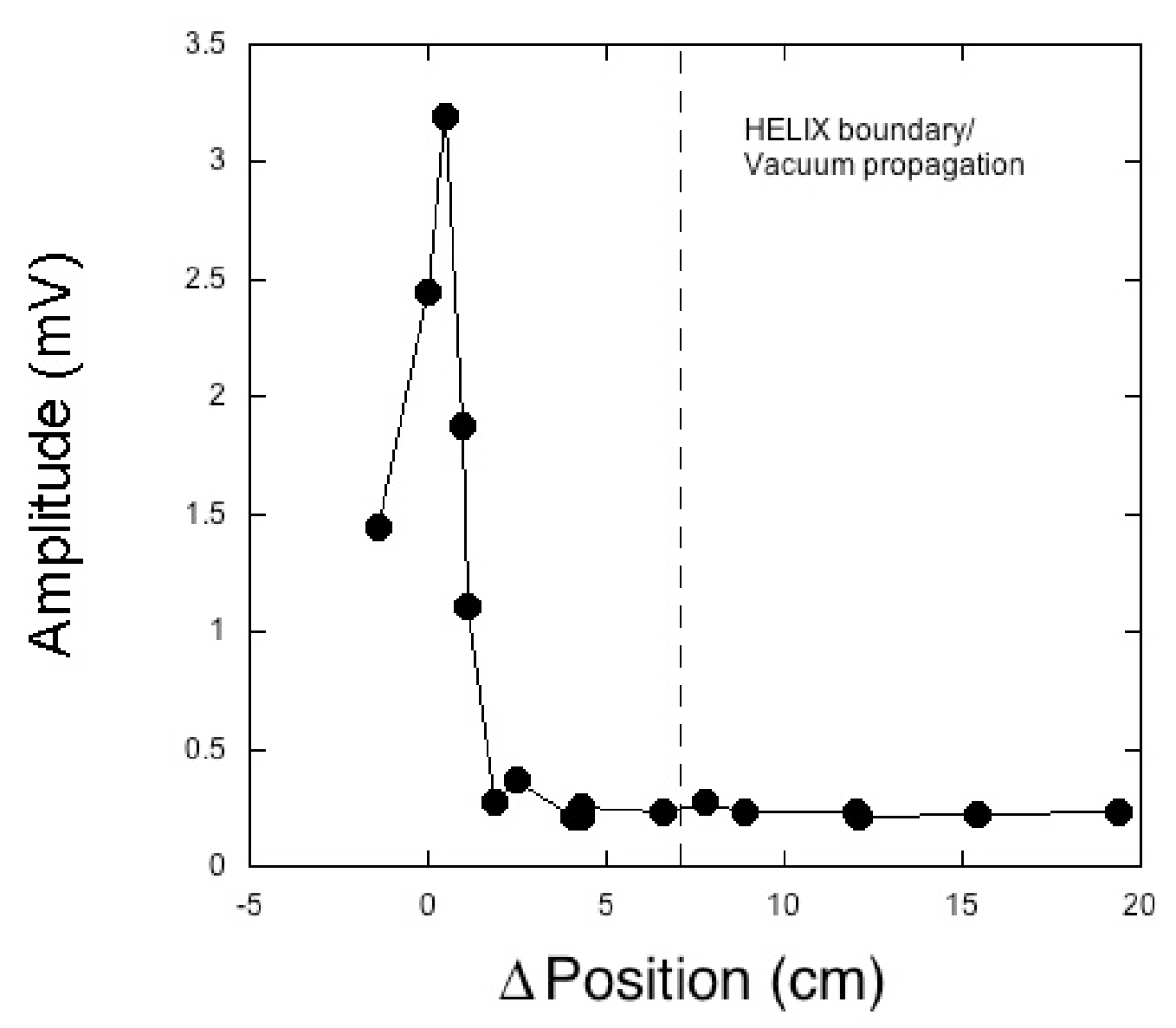

Figure 8. Amplitude of excited Alfvén wave in helium versus radial separation of the transmitter and receiver.

As noted previously, a fixed wave frequency of $112 \mathrm{kHz}$ was used while the ion cyclotron frequency was varied from $76.3 \mathrm{kHz}$ to $305.7 \mathrm{kHz}$ by varying the magnetic field from 200 Gauss to 800 Gauss. The dispersion relation (shown in Figure 9) obtained from this set of experiments did not agree with the theoretical dispersion relation. The disagreement between the experimental observation and the theory is likely due to the significant changes in the plasma parameters, e.g., plasma density, as the magnetic field strength was varied. Therefore, the Alfvén speed varied throughout the scan over magnetic field strength. As expected, for excitation frequencies close to the ion cyclotron 
frequency, the parallel wave number becomes large. The best curve fit available from the Kaleidagraph ${ }^{\circledast}$ software for these data produces an Alfvén speed of $1.6 \times 10^{6} \mathrm{~m} / \mathrm{s}$ which gives a wavelength of $14.2 \mathrm{~m}$, implying a half-wavelength standing wave inside the $7 \mathrm{~m}$ long chamber for a launching frequency of $112 \mathrm{kHz}$.

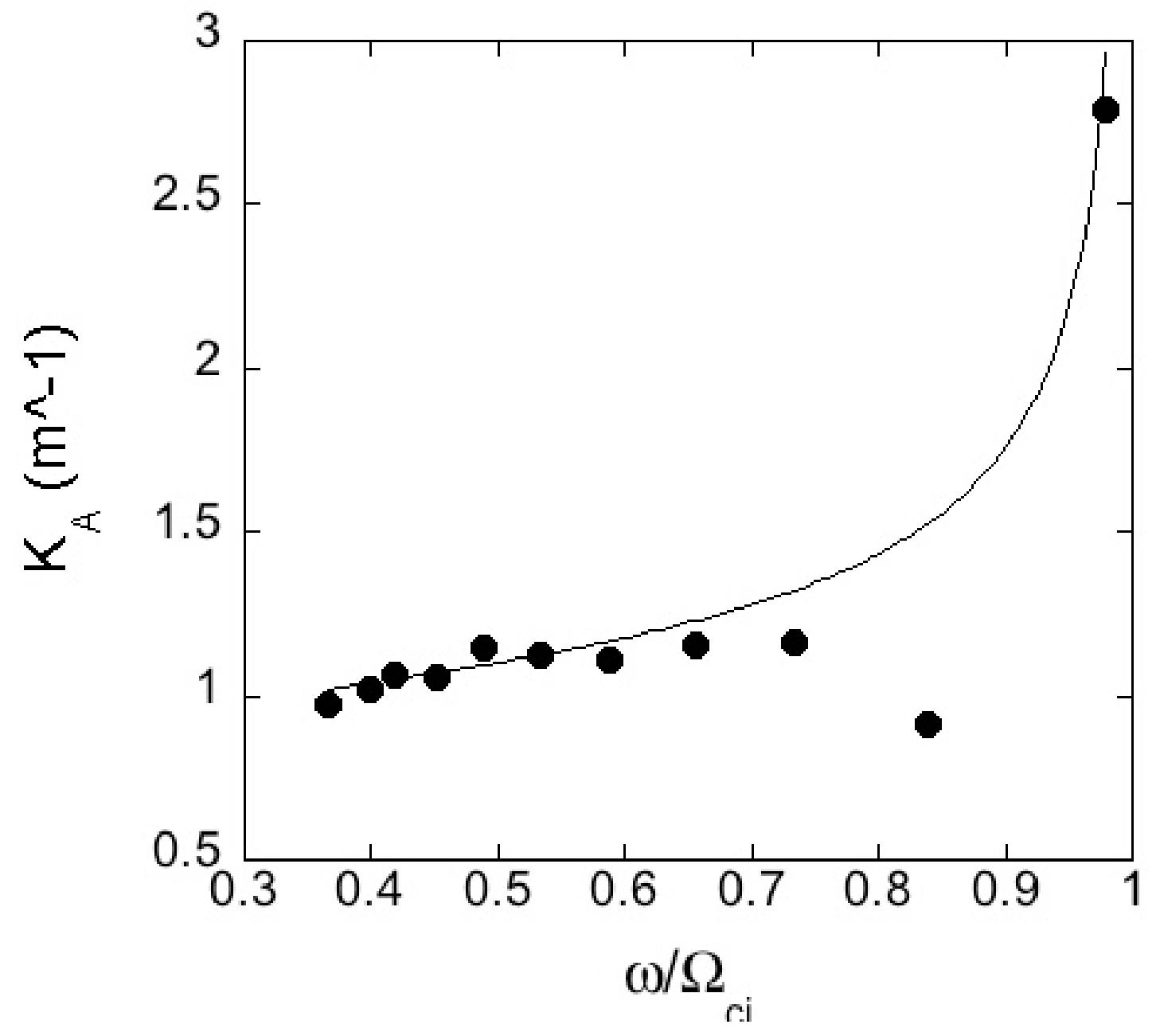

Figure 9. Parallel wave number versus normalized wave frequency $30.5 \mathrm{~cm}$ downstream in a helium HELIX plasma. Each phase (wave number) measurement was obtained at a fixed excitation frequency for different values of the magnetic field strength, i.e. the ion cyclotron frequency. The solid line indicates the curve fit for the data. 


\subsection{ARGON: FIXED FIELD, CHANGING DRIVE FREQUENCY}

The final set of measurements was carried out in argon plasmas at similar parameters to that of the first set of measurements in HELIX. The plasma source was operated at a pressure of $6.6 \mathrm{mTorr}$, RF power of 750 Watts, and a background magnetic field of 770 Gauss. These data were obtained following the replacement of HELIX magnet power supplies, which eliminated some noise in the measurements. The function generator was varied from $19 \mathrm{kHz}$ to $31 \mathrm{kHz}$ for an ion cyclotron frequency of $29.4 \mathrm{kHz}$. The result, shown in Figure 10, was obtained by measuring the phase between the receiving coil signal and the transmitter with a lock-in amplifier and subtracting off the phase lag due to the electronics. Since the lock-in amplifier was used, the differential amplifier was not necessary. From the dispersion relation a fit was made and the plasma

density determined to be $7.2 \times 10^{12} \mathrm{~cm}^{-3}$; consistent with previous density measurements at these parameters. At a fixed frequency of $19 \mathrm{kHz}$ this corresponds to an Alfvén speed of $9.9 \times 10^{4} \mathrm{~m} / \mathrm{s}$ and a wavelength of 5 meters. The perturbed field detected was 0.6 mGauss, or $7.8 \times 10^{-5} \%$ of the background field. 


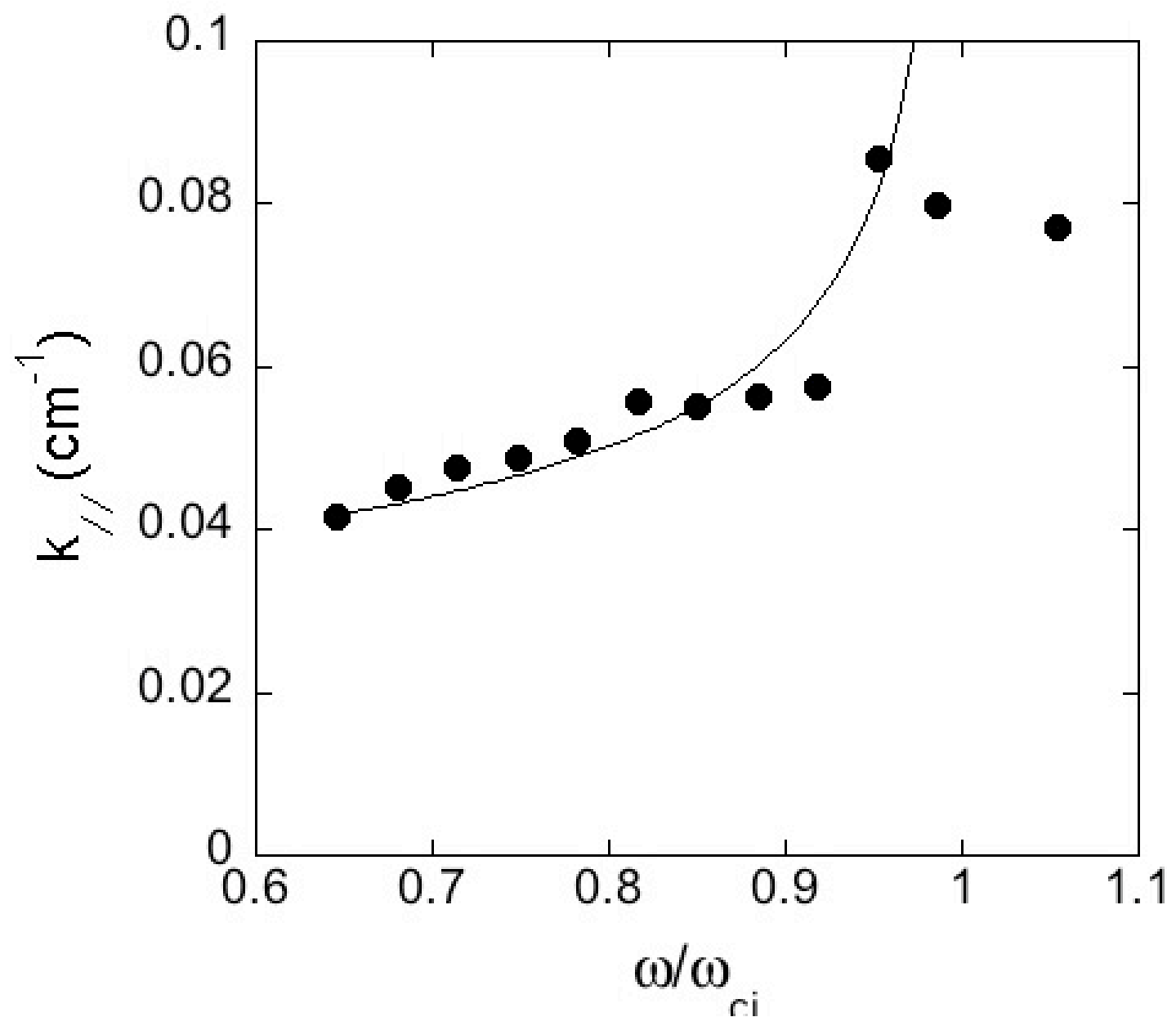

Figure 10. Parallel wave number versus normalized wave frequency $30.5 \mathrm{~cm}$ downstream in an argon HELIX plasma. Each phase (wave number) measurement was obtained at different excitation frequencies for a fixed value of the magnetic field strength. The solid line is a curve fit for the Alfvén wave resulting in a density of $7.2 \times 10^{12} \mathrm{~cm}^{-3}$

The wave amplitude radial profile shown in Figure 11 demonstrates that the propagating Alfvén wave is confined to a narrow region across the magnetic field. In this experiment, the plasma is operated in the kinetic regime $\left(v_{e} / v_{A}\right)^{2}=71.6$ rather than the 
inertial regime, $\left(v_{e} / v_{A}\right)^{2}<1$, as in the previous experiment. For this, a different theory is necessary to describe the radial profile measured in the device. Theory developed by Morales and Maggs [1997] shows a maximum angle of propagation, analogous to the cone angle in the inertial regime, given by,

$$
\tan \theta_{M}=\frac{\omega}{\omega_{c i}} \frac{a}{v_{A 0}}(1.1)
$$

where $v_{A 0}=c\left(\omega_{c i} / \omega_{p i}\right)$, and $\omega_{p i}=n e^{2} / \varepsilon_{0} m_{i}$ is the ion plasma frequency, where $n$ is the plasma density, $e$ is the electric charge, $\varepsilon_{0}$ is the permittivity of free space and $m_{i}$ the ion mass. The Alfvén wave propagation region is expected to become hollow at $16 \mathrm{~cm}$ downstream of the transmitter. This expression predicts that a hollow cone area will be produced that extends radially with a diameter of $1.26 \mathrm{~cm}$ from the $r=0$ position of the transmitter for our plasma parameters. At a radial position of $1.5 \mathrm{~cm}$ for the receiving coil, a sharp increase in signal amplitude is seen in Figure 11. The radial profile shows an increase in amplitude beginning at $1.5 \mathrm{~cm}$ and extending to $3 \mathrm{~cm}$ with the peak amplitude at $2.25 \mathrm{~cm}$. These measurements are generally consistent with propagation of the kinetic Alfvén wave at the maximum propagation angle for these parameters. 


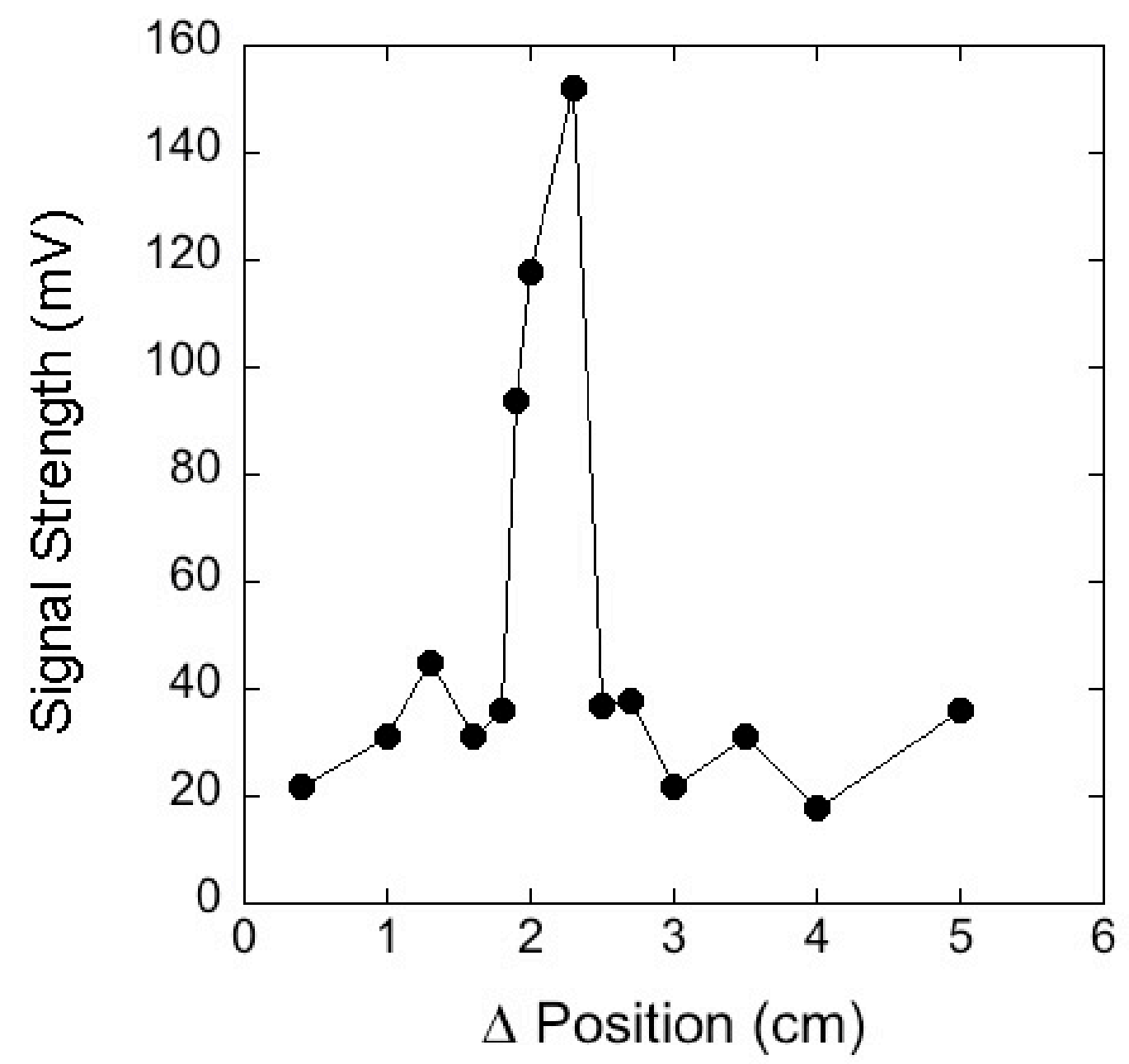

Figure 11. Amplitude of excited Alfvén wave in argon versus radial position of the receiver with the transmitter located at $0 \mathrm{~cm}$.

\section{CONCLUSIONS}

These measurements demonstrate that Alfvén waves may be reliably launched and detected in argon and helium HELIX plasmas. Detection of the low amplitude signals requires the use of appropriate amplifiers and knowledge of the location of the Alfvén cone at the receiving position. Future work will require a larger perturbing field, requiring a more robust coil capable of surviving a larger amplitude driving wave. 


\section{REFERENCES}

Alfvén, H., Nature 150, 405 (1942).

Bostick, W.H., and M., Levine, Phys. Rev. 87, 671 (1952).

Boyd and Sanders, The Physics of Plasmas, Cambridge University Press (2003)

Bloomburg, L.G. and J.A. Cummock, Adv. Space Res.., 33, 2161 (2004)

Cross, Rodney (1988) An Introduction to Alfvén Waves, Adam Hilger and IOP, Bristol, England

Chaston, C.C., et al., Phys. Rev. Lett., 95, 065002 (2005).

Colgate, S., J. Geophys. Res., 70, 3161 (1965).

Gekelman, W., et al., Phys. Plasmas 1, 3775 (1994).

Gekelman, W., et al., J. Geophys. Res. 108, A7, 1281 (2003).

Goldstein, M.L. et al., Annu. Rev. Astron. Astrophys. 33, 283 (1995).

Grulke, O., et al., $11^{\text {th }}$ International Congress on Plasma Physics 669, 412 (2002).

Hannah, J., and C. Watts, Phys. Plasmas 8, 4251 (2001)

Lundquist, S., Phys. Rev. 76, 1805 (1949)

Marsch, E., Physics of the Inner Heliosphere, Vol. 2, Springer (1991).

Maggs, J.E. and G.J. Morales, Phys. Rev Lett. 91, 3 (2003).

Mattheus, W.H, et al., Astrophys J. 523 L93 (1999).

Morales, G.J, J.E. Maggs, Phys. Plasmas 4, 4118 (1997)

Scime, E., et al., Rev. Sci. Instrum.73, 1970 (2002). 